\title{
Frontières
}

\section{La résilience et le deuil}

\section{Hélène Lefebvre et Bernard Michallet}

Volume 22, numéro 1-2, automne-printemps 2009-2010

Résilience et deuil

URI : https://id.erudit.org/iderudit/045020ar

DOI : https://doi.org/10.7202/045020ar

Aller au sommaire du numéro

Éditeur(s)

Université du Québec à Montréal

ISSN

1180-3479 (imprimé)

1916-0976 (numérique)

Découvrir la revue

Citer ce document

Lefebvre, H. \& Michallet, B. (2009). La résilience et le deuil. Frontières, 22(1-2),

7-9. https://doi.org/10.7202/045020ar

Ce document est protégé par la loi sur le droit d'auteur. L'utilisation des services d'Érudit (y compris la reproduction) est assujettie à sa politique d'utilisation que vous pouvez consulter en ligne.

https://apropos.erudit.org/fr/usagers/politique-dutilisation/
Cet article est diffusé et préservé par Érudit.

Érudit est un consortium interuniversitaire sans but lucratif composé de l’Université de Montréal, l’Université Laval et l'Université du Québec à Montréal. Il a pour mission la promotion et la valorisation de la recherche. https://www.erudit.org/fr/ 


\section{LA RÉSILIENCE ET LE DEUIL}

\section{Hélène Lefebvre et Bernard Michallet}

Le Groupe interréseaux de recherche sur l'adaptation de la famille et son environnement (GIRAFE), membre du Centre de recherche interdisciplinaire en réadaptation (CRIR), est fier de présenter dans le cadre du dixième anniversaire du CRIR ce numéro sur le thème de la résilience et du deuil.

La mission du CRIR est de contribuer, par la recherche dans les domaines biomédical et psychosocial, à l'autonomie et à l'intégration sociale des personnes ayant une déficience physique.

Le GIRAFE ${ }^{1}$ est constitué de chercheurs de milieux universitaires et de cliniciens provenant de centres de réadaptation en déficience physique. Ses activités de recherche et de transfert des connaissances portent depuis vingt ans sur le partenariat défini comme l'association entre une personne ayant des incapacités, ses proches, les intervenants, les gestionnaires, les décideurs du système de santé et les chercheurs dans un rapport visant l'égalité afin de mieux soutenir la participation sociale des personnes ayant des incapacités et leur famille. Chacun reconnaît l'expertise des autres et leurs ressources dans la prise de décision par consensus concernant la réalisation du projet de vie de la personne. Ce partenariat s'actualise dans un travail de coopération favorisant l'appropriation des compétences nécessaires pour mener à l'autodétermination de chacun (Bouchard, 1999)2 ${ }^{2}$. Le groupe est soucieux de codévelopper avec chaque partenaire des connaissances et des pratiques qui favorisent l'adaptation et l'intégration des personnes vivant avec une incapacité et leur famille. Ainsi, la reconnaissance des forces de la personne en interrelation avec les facteurs personnels et environnementaux peut aider la personne à s'adapter et même à transcender un événement ou une situation difficile. Ainsi, la résilience oblige à dégager les facteurs de réussite qui peuvent expliquer le succès malgré un sombre pronostic.

Dans la foulée de ses travaux, le GIRAFE s'intéresse à la résilience à la fois comme objet de recherche et de développement d'interventions innovantes en réadaptation. La résilience est un phénomène humain qui s'est fait de plus en plus présent dans les écrits à partir des années 1960, notamment à la suite des malheureuses expériences des camps de concentration et d'extermination où des personnes ont survécu à des conditions de vie extrêmes. Par la suite, elle a poursuivi son développement grâce aux travaux des éthologistes, des psychologues et des professionnels du domaine de la santé, des sciences humaines et de l'éducation. En réadaptation, la résilience devient une dimension incontournable tant pour l'intervention que pour la recherche.

Ce numéro propose un itinéraire qui retrace l'origine et l'évolution du concept de résilience ainsi que les diverses définitions que l'on retrouve dans les écrits. Il traite aussi bien de la résilience individuelle que familiale et communautaire. Bien que relativement peu d'études empiriques aient traité de la résilience, plusieurs chercheurs nationaux et internationaux s'y intéressent actuellement, comme le montrent certains articles de ce numéro. Il présente aussi les réflexions de chercheurs et de cliniciens préoccupés par le codéveloppement et l'évaluation d'interventions de réadaptation favorisant le rétablissement, le développement de l'autonomie des personnes et leur résilience. Enfin, deux points de vue de personnes ayant réfléchi sur le sujet sont présentés ainsi qu'un témoignage sur le deuil et la résilience viennent clore ce numéro.

Comme l'indiquent les articles de Bernard Michallet et celui de Michel Hanus, la résilience est un concept complexe dont la définition ne fait pas consensus. Pour certains auteurs, elle est le résultat d'activités ou de capacités, pour d'autres, la résilience est un processus qui permet de transformer une expérience difficile en réussite de vie. Bernard Michallet, afin de concrétiser le phénomène, propose des considérations cliniques pour le domaine de la réadaptation physique. Pour Michel Hanus, résilience et deuil ont des points en commun dont leur origine traumatique mais comportent aussi des différences. Cet article jalonné d'exemples offre aussi une réflexion sur leur articulation. «Le temps, les temps de la résilience entretiennent des affinités avec ceux du deuil mais restent marqués par de grandes différences.» L'article de Patrick Fougeyrollas et Claire Dumont jette un nouvel éclairage sur le concept en suggérant que la résilience est présente lorsque la personne vit un processus de construction ou de reconstruction identitaire favorable en dépit des obstacles. Celle-ci peut 
être la cible de l'intervention de réadaptation. Une originalité de cet article consiste à décrire l'apport d'un cadre conceptuel, le Processus de production du handicap ( $\mathrm{PPH})$, à la compréhension de la résilience. De plus, Patrick Fougeyrollas fait une distinction entre le développement de la résilience chez une personne qui naît ou acquiert une différence potentielle en bas âge et celle qui subit une rupture traumatique qui laisse des incapacités organiques ou symboliques, physiques ou mentales. La résilience est vue ici comme le résultat d'une interaction entre les dimensions organiques, psychologiques et fonctionnelles, la qualité de l'environnement physique et social et les habitudes de vie. Comme Bernard Michallet et Michel Hanus le proposent, Patrick Fougeyrollas renchérit sur l'importance de vivre des succès, de proposer des modèles favorables et d'être en contact avec un environnement enrichissant; ces éléments peuvent contribuer à la résilience.

Yves Boisvert invite le lecteur à entrer dans l'univers d'une personne présentant une incapacité intellectuelle. Dans ce contexte particulier, il envisage la résilience sous l'angle de la trajectoire de vie dans une perspective interactive entre les facteurs de risque et les facteurs de protection. Cet article s'attarde à donner un sens historique à la relation d'accompagnement, puis un sens théorique en intégrant le concept de résilience dans une adaptation clinique du Processus de production du handicap en déficience intellectuelle; et enfin un sens clinique en proposant l'élaboration d'une philosophie d'intervention comportant des grands principes à considérer lorsque la résilience devient un concept central de la pratique professionnelle en déficience intellectuelle.

Pour Josiane Crête, qui aborde l'adaptation et la résilience des familles à la suite d'un événement traumatisant, la résilience est une des issues possibles du processus d'adaptation des proches de personnes ayant une déficience physique après un processus de deuil. Pour elle, le processus d'adaptation comporte quatre étapes chronologiques: la période avant l'annonce du diagnostic, le choc suivant cette annonce, la période de réadaptation et celle après la réadaptation. S'appuyant sur le modèle de Richardson et ses collaborateurs, elle décrit comment le système familial peut se transformer en donnant un sens constructif à la situation vécue.

Christiane Couture, dans son article sur l'adaptation et la résilience dans le cas d'une maladie dégénérative, la sclérose en plaques, insiste sur la quête de sens malgré les deuils récurrents. Après avoir décrit le processus d'adaptation à travers des théories sur le deuil, elle montre comment une intervention de groupe «Vivre en déséquilibre» auprès de ces personnes peut favoriser une adaptation réussie entraînant la résilience par la recherche d'un sens positif à l'expérience.

De leur côté, Mélanie Dumais, Audrey Fortin et Denise Beaudry offrent une activité d'expression musicale en centre de réadaptation pour cette clientèle et leurs proches afin de favoriser le processus de résilience. Elle a pour but de faciliter l'émergence de stratégies d'action pour réagir à la maladie. Selon les études citées, la musique apporterait des changements positifs d'humeur en neuroréadaptatation. Cet article décrit entre autres le déroulement de cet atelier d'expression musicale et les enjeux psychosociaux de l'intervention.

Diane Pelchat présente les résultats d'une étude qualitative réalisée auprès de pères et de mères d'enfants ayant une déficience. Ceux-ci montrent des différences entre les parents dans l'expérience de deuil de l'enfant attendu, lesquelles semblent porteuses de transformation par leur caractère complémentaire. Cette étude s'appuie sur un modèle clinique du processus d'adaptation/ transformation.

L'article de Christine Genest et de Francine Gratton présente une analyse de concept sur la résilience familiale afin d'en avoir une meilleure compréhension. Par la suite, le deuil lié au suicide d'un adolescent est décrit en soulignant son caractère particuliè- rement éprouvant. Comme le rapportent les auteures, malgré une telle perte, les familles survivent et poursuivent leur développement. Toutefois, peu d'études ont décrit la résilience familiale. Une étude est actuellement en cours auprès de familles endeuillées à la suite du suicide d'un adolescent afin de décrire le processus de résilience familiale dans ce contexte.

Marilou Parser et Monique Séguin présentent les résultats d'une étude exploratoire à devis mixte (quantitatif et qualitatif) portant sur la participation de personnes en processus de deuil à la suite du suicide d'un proche. Elles tentent de répondre à la question: les personnes en deuil peuvent-elles tirer profit d'une participation

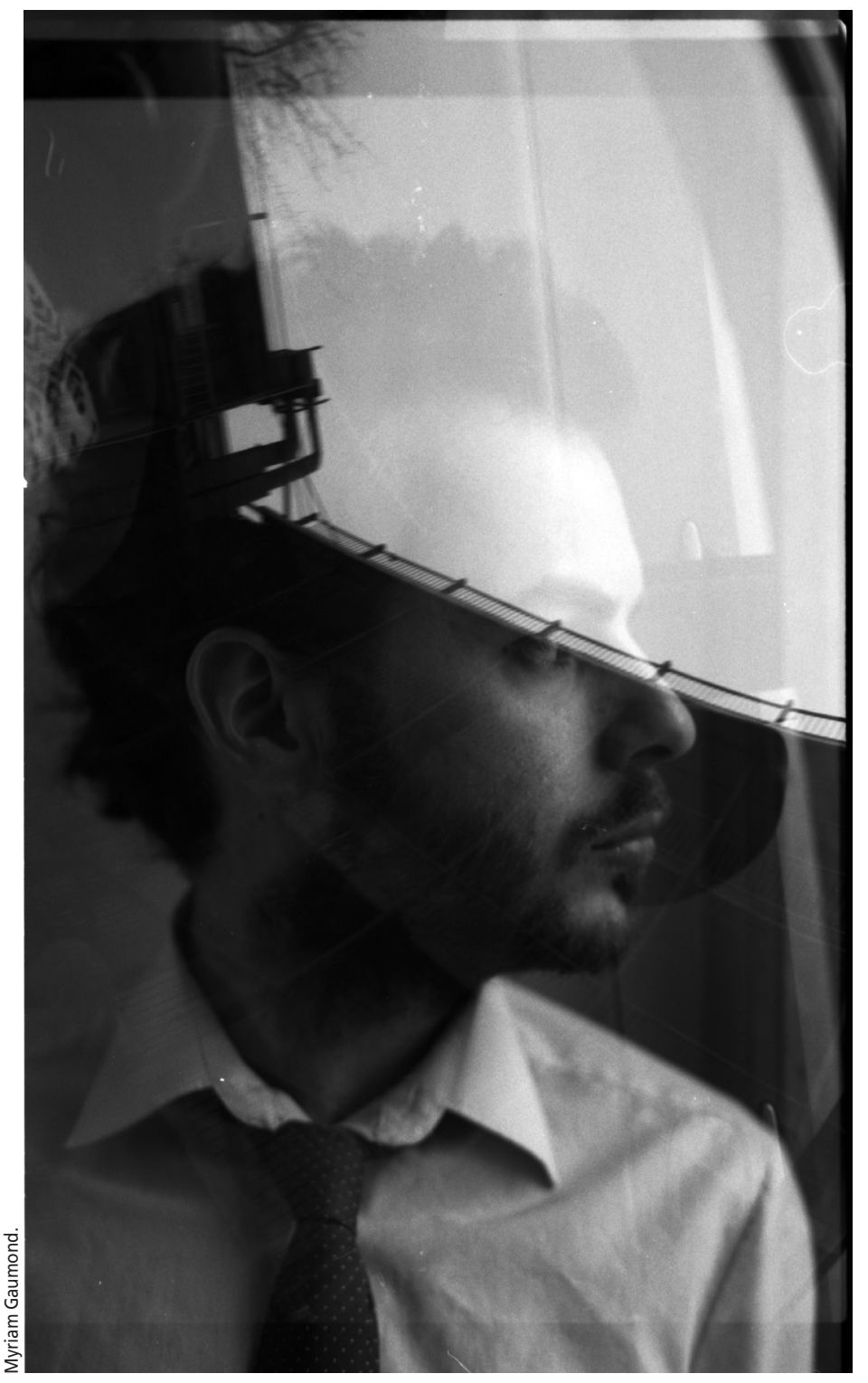

à une recherche? Cette étude compare les perceptions de deux groupes de personnes ayant participé à une recherche alors qu'elles étaient en situation de vulnérabilité, soit des personnes endeuillées interviewées immédiatement après le processus de recherche et un second groupe, une année après celui-ci. Les résultats des deux groupes sont semblables, et tous les participants accepteraient de recommencer l'expérience et recommanderaient à d'autres personnes endeuillées de participer à une telle étude.

L'article de Jan Gelech et Michel Desjardins rapporte les résultats d'une étude qualitative menée auprès de personnes ayant subi un traumatisme crânien et ayant pour objectifs l'identification de stratégies de reconstruction de leur monde vécu et de leur personne. Du point de vue de la théorie des rites de transition 
développée par Victor Turner, ces stratégies sont: le réalignement, la transcendance et l'intégration dans des marges. Les auteurs terminent leur analyse en proposant une série de questions en projection vers l'avenir de ces personnes, par exemple: "Au total, l'inclusion accroîtra-t-elle ou diminuera-t-elle leur capacité de se lier aux autres, d'appartenir à une collectivité, d'affirmer leur dignité, de se dépasser, d'aimer la vie, de se projeter dans l'avenir et de donner un sens à leur existence? »

Hélène Lefebvre, Marie-Josée Levert et Jérôme GauvinLepage décrivent une intervention personnalisée d'accompagnement dans la communauté de personnes ayant un traumatisme

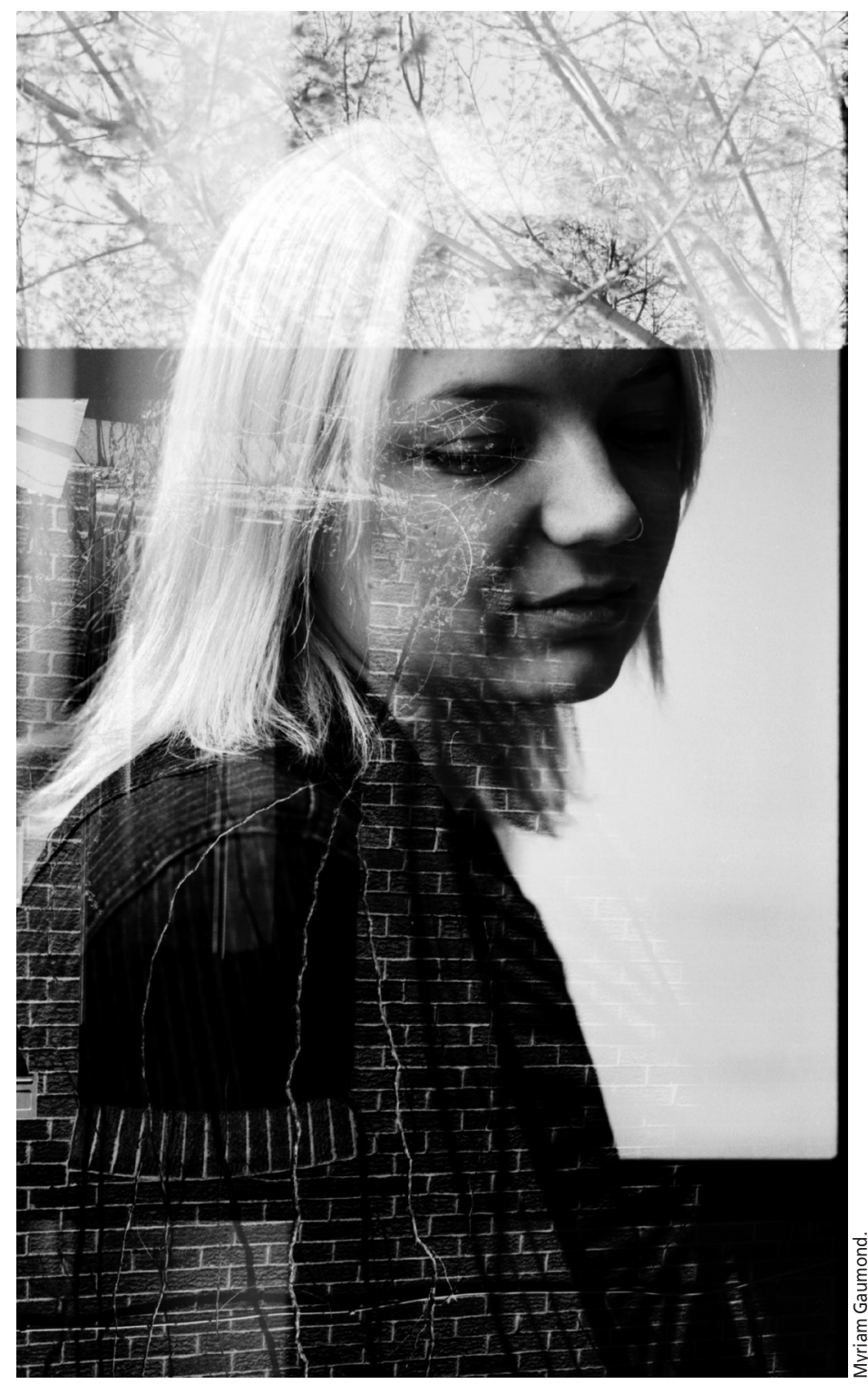

Frontières présente dans ce numéro des œuvres de Myriam Gaumond.

« Myriam Gaumond est une photographe émergente de Montréal, travaillant sur le concept de photographe-observateur. Ses œuvres traitent de l'inévitable subjectivité de la création photographique. Son travail provoque autant des réflexions sur l'individualité et la solitude que sur la vie publique et la place du photographe dans celleci. Ces observations photographiques se font toujours dans un contexte évoquant une intimité et une poésie simple et délicate. " (Extraits du texte de présentation de l'artiste, www.visualvoicegallery.com).

Certaines des œuvres présentées dans ce numéro sont tirées de la série Surimpressions. La galerie d'art contemporain Visual Voice, à Montréal, a présenté des œuvres de Myriam Gaumond en 2007 ainsi que lors d'une exposition solo en 2009. Myriam Gaumond est présentement inscrite à la majeure en photographie, à I'Université Concordia.

Site Internet de l'artiste : <http://www.myriamgaumond.com> crânien modéré à sévère qui sont retournées vivre à domicile sans services en réponse à leurs besoins. L'IPIC est en cours d'implantation et d'évaluation. Elle vise le maintien dans le milieu de vie de ces personnes en favorisant leur participation sociale et en soutenant le développement de leur résilience. Pour cela, elle s'appuie sur l'utilisation des caractéristiques positives individuelles, familiales et communautaires, pour faciliter l'appropriation de savoirs et d'habiletés en vue de favoriser une meilleure autodétermination et un soutien au développement d'habiletés relationnelles et familiales.

L'article de Jocelyn Chouinard porte sur la dimension spirituelle du concept de résilience. Après avoir résumé les définitions du concept de résilience et celle de spiritualité, il s'appuie sur le postulat de l'école de pensée de la psychologie positive selon lequel «la personne est capable d'agir sur son bien-être et sur sa santé». Cet auteur affirme que «le défi est de comprendre comment une situation de handicap peut se transformer en une situation de développement et de croissance à la suite de "l'interaction dynamique de facteurs appartenant à l'individu et à l'environnement" ». Ainsi, le défi de l'intervention de réadaptation est d'amener la personne à travers son expérience personnelle à découvrir un sens à l'événement traumatique vécu afin de faire de celui-ci une expérience de transformation et d'actualisation qui sera un point tournant de son projet de vie. C'est dans cet esprit que la méthode d'intervention «le Fil d'Ariane» a été développée. Jocelyn Chouinard présente l'histoire de Mathilde pour illustrer l'utilisation de celui-ci.

Edouardo Cisneros et ses collègues présentent un instrument clinique qui favorise l'auto-observation et l'autoévaluation du degré de fonctionnement de la personne dans la réalisation de ses habitudes de vie - l'outil interdisciplinaire Priorités d'intervention du client (PIC). Le PIC est basé sur l'accomplissement des habitudes de vie et la sélection par la personne de priorités d'intervention sur le plan de la réadaptation. Il concrétise une approche axée sur la personne, sur le respect de son individualité et de son intimité. Celui-ci en stimulant la motivation de la personne à s'engager dans son processus de réadaptation devrait aussi contribuer au développement de sa résilience.

Lucille Guilbert présente un article sur la médiation culturelle des ressources symboliques et narratives dans le processus de résilience qui est au cœur de l'expérience migratoire. À travers quatre exemples de «dits de vie», cet article propose des pistes d'utilisation quant à l'élaboration collective de dits de vie en groupes interculturel issus de récits de tradition orale, de récits de rêves, ou de textes littéraires qui constituent des ressources symboliques de résilience dans le parcours des personnes immigrantes et réfugiées. Ces dits de vie sont issus de l'expérience des personnes réfugiées que l'auteur a rencontrées dans un camp de réfugiés en Thaïlande, lors d'un séjour en Croatie, et depuis 1995 à Québec dans le contexte d'accueil et d'accompagnement de personnes réfugiées de Bosnie-et-Herzégovine, d'Afghanistan, du Kosovo, de Colombie, et d'ateliers de rapprochement interculturel et de formation d'intervenants.

Pour clore ce parcours, les témoignages de Chloé Sainte-Marie, dans une entrevue avec Aimée Verret, de Bernard Lefebvre et de Émilie C. Lévesque nous rappellent chacun à leur façon que la résilience et le deuil se vivent au quotidien.

Bien que non exhaustif, mais non sans ambitions, voilà le tour d'horizon que propose ce numéro.

\section{Notes}

1. 〈http://www.crir.ca/girafe>.

2. J.-M. BOUCHARD (1999). «Famille et savoirs à partager: des intentions à l'action », Apprentissage et socialisation, vol. 19, n 2, p. 47-57. 